\title{
Towards universal ARV access: Achievements and challenges in Free State Province, South Africa
}

\author{
Kerry E Uebel, Venessa Timmerman, Suzanne M Ingle, Dingie H C J van Rensburg, Willie F Mollentze
}

Objective. To study the progress and challenges with regard to universal antiretroviral (ARV) access in Free State Province, South Africa.

Methods. Data from the first 4 years of the public sector ARV roll-out and selected health system indicators were used. Data were collected from the public sector ARV database in Free State Province for new patients on ARVs, average waiting times and median $\mathrm{CD} 4$ counts at the start of treatment. Information on staff training, vacancy rates and funding allocations for the ARV roll-out was obtained from official government reports. Projections were made of expected new ARV enrolments for 2008 and 2009 and compared with goals set by the National Strategic Plan (NSP) to achieve universal access to ARVs by 2011.
Results. New ARV enrolments increased annually to $25 \%$ of the estimated need by the end of 2007. Average waiting times to enrolment decreased from 5.82 months to 3.24 months. Median CD4 counts at enrolment increased from 89 to 124 cells/ $\mu l$. There is a staff vacancy rate of $38 \%$ in the ARV programme and an inadequate increase in budget allocations.

Conclusion. The current vertical model of ARV therapy delivery is unlikely to raise the number of new enrolments sufficiently to achieve the goals of universal access by 2011 as envisaged by the NSP. The Free State is implementing a project (STRETCH trial) to broaden the ARV roll-out in an attempt to increase access to ARVs.

S Afr Med J 2010; 100: 589-593.
South Africa, with an estimated 5.7 million people living with HIV, has the largest burden of HIV disease globally. ${ }^{1}$ By 2009, an estimated 2.9 million South Africans had died of AIDS, with an annual AIDS mortality of $374000 .^{2}$ The Free State (FS), one of 9 provinces in South Africa, with an estimated population of $2792000,{ }^{2}$ has an AIDS prevalence rate of $18.5 \%$ among 15 - 49 -year-olds, compared with the national figure of $16.9 \%{ }^{3}$

To combat the social disaster in high-burden countries, calls were made in 2003 to start the formidable task of getting antiretrovirals (ARVs) to people who needed them. ${ }^{4}$ By the end of 2008, the World Health Organization (WHO) estimated that over 4 million people had accessed ARVs in developing countries. In South Africa, specifically,

Knowledge Translation Unit, UCT Lung Institute, Cape Town, and Department of Internal Medicine, Faculty of Health Sciences, University of the Free State, Bloemfontein

Kerry E Uebel, BSc (Med), MB BS, MFamMed

Knowledge Translation Unit, UCT Lung Institute, Cape Town Venessa Timmerman, BSc, MSc

Department of Social Medicine, University of Bristol, UK Suzanne M Ingle, BSc, MSc

Centre for Health Systems Research and Development, University of the Free State, Bloemfontein

Dingie H C J van Rensburg, MA, DPhil

Department of Internal Medicine, Faculty of Health Sciences, University of the Free State, Bloemfontein

Willie F Mollentze, MD, FCP (SA), MMed (Int Med), FACE
700500 people were reported to be accessing ARVs. ${ }^{5}$ UNAIDS has published estimates of the total need for ARVs in different regions, defined as the number of people accessing ARVs plus those who qualify for ARVs, i.e. either with stage 4 disease (AIDS) or a CD4 count $<200$ cells $/ \mu$ l. Using this definition, an estimated $42 \%$ of people in developing countries who need ARVs are accessing them. ${ }^{5}$

In 2003, the South African National Department of Health (DOH) launched the national ARV roll-out with its Comprehensive Plan for the Care, Management and Treatment of HIV and AIDS (Operational Plan). Its aim was to achieve universal ARV access (an estimated 1.4 million people in need of ARVs at that stage) within 5 years. ${ }^{6}$ Although the OP's stated aim was to provide comprehensive HIV care in an integrated fashion, the roll-out was delivered as a vertical programme with dedicated funding, staffing and administration, and closely controlled national accreditation of ARV sites. ${ }^{6}$

International debate continues on the merits of horizontal versus vertical programmes for delivering HIV care in developing countries. The advantage of vertical (stand-alone) programmes is their ability to deliver rapid roll-out of the type of complex health intervention needed to tackle HIV. ${ }^{7}$ However, it is thought that such vertical programmes will not be able to achieve universal access and that they need to be broadened using the new so-called diagonal approach. ${ }^{8}$ By 2006 it was clear that universal access to ARVs in South Africa would take more than 5 years to achieve. Less than $30 \%$ of those in need were accessing ARVs. ${ }^{5}$ In 2007, the DOH published its National Strategic Plan (NSP) with detailed annual goals, aiming to achieve access to ARVs for $80 \%$ of the people who would need them by $2011,{ }^{9}$ and defining the need for ARVs as the number of new AIDS patients, estimated at about 520000 annually, from 2007 to $2011 .{ }^{3}$ In contrast with the WHO definition of need for ARVs, the former was an annual figure against which one can measure the number of new patients accessing ARVs each year, but it does not include estimates of people with a CD4 count below 200 cells/ $\mu$ land therefore probably underestimates need.

In the FS, the ARV roll-out began in mid-2004 as a vertical programme with plans to establish ARV sites in each of its 20 local areas. ${ }^{10}$ Two types of ARV sites were established. Treatment sites were led by doctors, where patients were referred for initiation and monitoring of ARVs. These were linked to nurse-led assessment sites 
in primary health care (PHC) facilities, where patients would be screened and prepared for ARVs and be able to collect medication. This model was adapted particularly for small-town sites with the accreditation of combined sites, i.e. a nurse-led assessment site with doctors visiting regularly. Reviews of the FS roll-out noted that the vertically run ARV programme drained staff from other PHC facilities and that problems were experienced with integration of ARV care into PHC services. ${ }^{11}$

Our study has lessons for achieving universal access to ARVs in high-burden countries. Our objective was to attempt to answer two questions: (i) what is the progress towards universal ARV access in the FS in the first 4 years of the roll-out; and (ii) how do the challenges in the FS inform the debate on the merits of vertical versus horizontal delivery systems in achieving universal ARV access in high-burden countries?

\section{Methods}

\section{Study design and data collection}

A retrospective, observational study was conducted using data from a variety of sources. Information on the number of ARV sites established in the FS from May 2004 until June 2008, expenditure, and staff vacancy rates for the ARV programme was obtained from the province's Directorate: Comprehensive HIV and AIDS Management. Information on conditional grant allocations was obtained from the National Treasury website. ${ }^{12}$ Data on patients initiated on ARVs were obtained from the FS ARV database. Routine clinical data from all public sector ARV sites in the FS (with the exception of 3 small-town sites not yet online) were entered on an electronic record system (Meditech) by clinic-based data capturers. The data were incorporated in a data warehouse and used for monitoring and evaluation. This routinely collected dataset was supplemented by linkage to the National Health Laboratory Service (NHLS) database. Information on the estimated annual number of new AIDS patients in the FS was retrieved from the Actuarial Society of Southern Africa's 2003 model of demographic indicators for the HIV epidemic. ${ }^{13}$

Permission to conduct this study was granted by the Head of the FS Department of Health. The Ethics Committee of the Faculty of Health Sciences, University of the Free State, approved the protocol.

\section{Staff training}

The FS Department of Health runs a regular 2-week ARV training course for medical, nursing and ancillary staff. Information on staff training was obtained from the province's Directorate: Human Resource Management, and from district managers. It has also implemented on-site training in integrated management of respiratory illnesses, tuberculosis (TB), HIV (including ARVs) and sexually transmitted infections (STIs) in adults, using the PALSA PLUS guidelines. ${ }^{14}$

\section{Statistical analysis}

The monthly new ARV enrolments from 2004 to 2007 were plotted and an extrapolation of a linear trend line was fitted to the data to project expected increases for 2008 and 2009 using statistical packages available in Microsoft Excel. Median waiting times for ARV initiation were calculated using Kaplan-Meier estimates. Waiting time was defined as time between first CD4 count $<200$ cells/ $\mu$ and initiation of ARVs. Patients who died before the start of treatment or who were not observed to start ARVs were censored in these analyses. Median $\mathrm{CD} 4$ counts at commencement of ARVs were calculated over 3-month periods. The significance of a trend over time in enrolment $\mathrm{CD} 4$ counts was checked using an extension of the Wilcoxon rank sum test. Survival analyses and tests for trend were done using Stata version 10.1.

\section{Results}

The number of all types of sites increased steadily from 20 sites in 6 local areas by the end of 2004, to 56 sites by August 2008, with at least 1 site in each of the 20 local areas. There were 11 treatment sites, 27 assessment sites and 18 combined sites.

The number of new patients (adults and children) commencing ARVs each month increased steadily, peaking at 1045 in October 2007 (Fig. 1). Comparing the annual number of patients starting ARVs with the estimated number of new AIDS patients for $2004-2007,{ }^{13}$ the percentage coverage increased from $2 \%$ in 2004 to $25 \%$ in 2007 (Table I). Patients were distributed unevenly across the 56 sites, with 7 urban sites each having initiated treatment on more than 1000 patients.

Should the expansion of the ARV roll-out continue as in the period 2004 - 2007, monthly new ARV enrolments for 2008 and 2009 could be expected to have increased as in the trend line in Fig. 1. According to this projection, about 11500 patients would have started ARVs in 2008 and about 14300 in 2009, representing about $31 \%$ and $39 \%$ of estimated new AIDS patients, respectively. ${ }^{13}$ These percentages would fall short of the goal of the NSP ${ }^{9}$ to increase enrolment of new patients on ARVs to 35\% of need in 2008 and 55\% in 2009 (Table II). If, however, NSP goals were achieved in the FS, the cumulative number of patients on treatment at ARV sites would more than double to reach approximately 44267 (Table II).

The median waiting times from eligibility (CD4 count $<200$ cells/ $\mu \mathrm{l})$ to starting ARVs decreased from 5.82 months in 2004 to 3.24 months in 2007 (Table III). The analysis of waiting times was

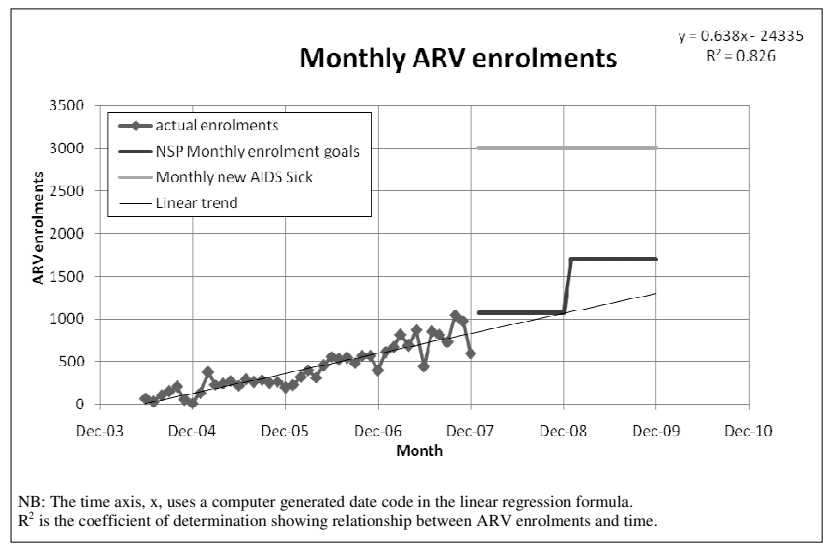

Fig. 1. Monthly new enrolments (2004) plotted against need and goals as envisaged in the National Strategic Plan 2008 and 2009.

Table I. Annual enrolments on ARVs compared with estimated new AIDS patients in the Free State $2004-2007$

\begin{tabular}{lcl}
\hline & New AIDS patients & $\begin{array}{l}\text { Total } \\
\text { enrolled on } \\
\text { ARVs }(\% \\
\text { of need) }\end{array}$ \\
\hline Year & 29104 & $642(2.2)$ \\
2004 & 32124 & $3091(9.6)$ \\
2005 & 34431 & $5420(15.7)$ \\
2007 & 36012 & $9157(25.4)$ \\
Total & 131671 & 18310 \\
\hline
\end{tabular}


Table II. Goals for new enrolments on ARVs for the Free State as envisaged in the National Strategic Plan (NSP)

\begin{tabular}{|c|c|c|c|c|c|}
\hline Year & $\begin{array}{l}\text { New AIDS } \\
\text { patients }\end{array}$ & $\begin{array}{l}\text { Monthly new } \\
\text { enrolments to } \\
\text { meet need }\end{array}$ & $\begin{array}{l}\text { Goals for new } \\
\text { enrolments } \\
\text { according to } \\
\text { NSP (\%) }\end{array}$ & $\begin{array}{l}\text { Monthly new } \\
\text { enrolments to } \\
\text { meet NSP goals }\end{array}$ & $\begin{array}{l}\text { Estimated } \\
\text { cumulative } \\
\text { numbers on } \\
\text { ARVs }^{*}\end{array}$ \\
\hline 2008 & 36861 & 3070 & $12901(35)$ & 1075 & 28360 \\
\hline 2009 & 37059 & 3090 & $20382(55)$ & 1700 & 44267 \\
\hline 2010 & 36737 & 3070 & $25716(70)$ & 2140 & 63912 \\
\hline 2011 & 36042 & 3000 & $28834(80)$ & 2400 & 85225 \\
\hline
\end{tabular}

Table III. Waiting time from eligibility to treatment for each calendar year and health district $(N=22496)^{*}$

\begin{tabular}{|c|c|c|c|c|c|c|c|c|}
\hline \multirow{3}{*}{ District } & \multicolumn{8}{|c|}{ Year of enrolment } \\
\hline & \multicolumn{2}{|c|}{2004} & \multicolumn{2}{|c|}{2005} & \multicolumn{2}{|c|}{2006} & \multicolumn{2}{|c|}{2007} \\
\hline & $N$ & MWT & $N$ & MWT & $N$ & MWT & $N$ & MWT \\
\hline Fezile Dabi & 363 & 6.84 & 734 & 3.04 & 883 & 3.44 & 769 & 3.04 \\
\hline Xhariep & 422 & 11.27 & 331 & 6.21 & 591 & 3.80 & 539 & 2.78 \\
\hline Motheo & 1624 & 6.02 & 1599 & 7.90 & 1126 & 5.02 & 2428 & 3.83 \\
\hline Lejweleputswa & 914 & 4.83 & 1146 & 3.50 & 1416 & 3.07 & 1331 & 2.84 \\
\hline Thabo Mofutsanyane & 676 & 4.86 & 1544 & 6.55 & 2210 & 6.28 & 1850 & 2.74 \\
\hline Total & 3999 & 5.82 & 5354 & 5.75 & 6226 & 4.36 & 6917 & 3.24 \\
\hline
\end{tabular}

repeated using cumulative incidence functions to account for the competing risk of patients dying before accessing treatment. This made no difference to the trend of median waiting times decreasing over time. Pre-ARV CD4 cell counts were available in the dataset for $75 \%$ of the 17847 patients who started ARVs by December 2007. Many CD4 results were not entered onto the database, particularly in 2006 and 2007, as workload increased and not all missing results could be matched from the NHLS database.

The median baseline $\mathrm{CD} 4$ cell counts at commencement of ARVs rose from 89 cells/ $\mu$ (interquartile range (IQR) 49 - 156) in the second quarter of 2004 to 124 cells/ $\mu$ (IQR 55 - 84) in the last quarter of 2007 (Fig. 2). There is strong evidence of a positive trend over time $(\mathrm{z}=7.71$ Prob $>|\mathrm{z}|=0)$.

Human resources and training. By June 2008, 534 posts had been created in the ARV programme, although 207 (38.8\%) were

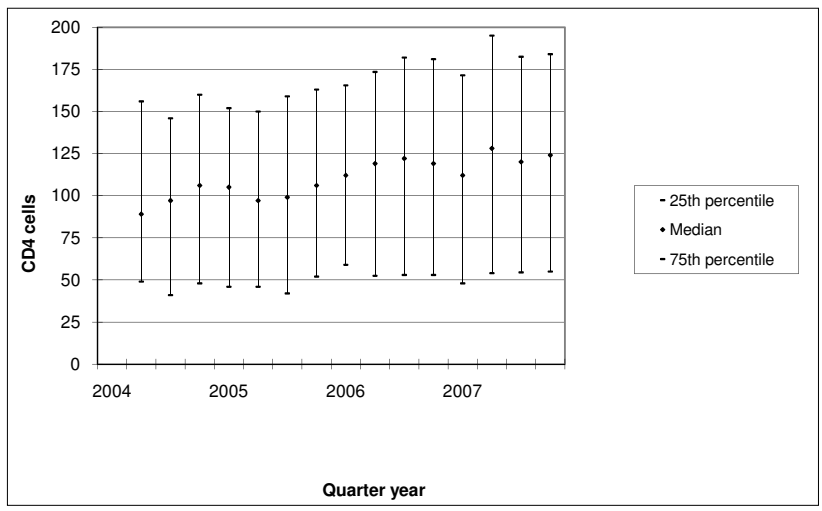

Fig. 2. Plot of median CD4 cell counts at initiation of $A R V s^{*}$ and their IQRs at each quarter, $\mathrm{N}=5$ 918. $\left({ }^{*}\right.$ Based on 5918 patients with a $C D 4$ result in the period 6 weeks before - 4 weeks after commencement of ARVs.) vacant. Of the professional posts, 39\% nurses', 50\% doctors' and 59\% pharmacists' posts were vacant.

Training of staff in the management of HIV including ARVs was not restricted to staff from the ARV programme. From May 2004 until August 2008, 2540 members of staff, including 251 doctors and 1130 professional nurses, had completed the 2-week ARV training course. At least 1 professional nurse from every PHC clinic in the province had been trained. By August 2008, staff in 139 of the approximately 240 PHC clinics had been trained in managing patients using the PALSA PLUS guidelines.

Funding for the ARV roll-out is provided by the DOH, with an annual allocation to the provinces of a conditional grant for comprehensive HIV and AIDS management. Amounts allocated to the FS have improved annually, with more than $40 \%$ increases over the first 2 years, a 24\% increase in 2007/2008 for the 2008/2009 financial year, and a $20 \%$ increase from $2008 / 2009$ to $2009 / 2010$. Of the R153 646000 allocated in the 2007/2008 conditional grant to the FS, $35 \%$ was spent on drugs and laboratory tests, and $39 \%$ on staff remuneration for full-time and lay workers in the ARV sites. The rest was spent on training, prevention programmes, infrastructure, stepdown care and programme management.

\section{Discussion}

The public sector ARV programme in the FS achieved steadily rising numbers of monthly new enrolments on ARVs and decreasing waiting times for those eligible for ARVs. CD4 counts were higher at commencement of ARVs, and ARV services were provided in all its 20 local areas. These findings suggest that the programme is making inroads into the shortfall, and people are accessing ARVs more readily. Published figures show good outcomes of patients receiving ARVs in the public sector in the FS, with a 7\% mortality rate and an $82 \%$ viral load suppression rate at 1 year. ${ }^{15}$ 
A strength of this study is the availability and completeness of data for patients before and during ARV initiation, supplemented by linkages with the NHLS database. A weakness as a retrospective study is that, because of an increasing data entry backlog, no accurate figures are available on patient retention in the ARV programme. Furthermore, these findings do not include data for patients on ARVs outside the public sector in the FS.

However, assuming that most people who need ARVs in the FS will only have access to public facilities, the percentage that accessed ARVs is small and the FS has a long way to go towards universal ARV coverage. From 2004 to 2007, only 18310 people were initiated on ARVs in the public sector (Table I), whereas an estimated 131671 developed AIDS in those 4 years. ${ }^{13}$ Even in 2007, ARV initiations reached only $25 \%$ of the estimated need. Presumably, many of those who did not start ARVs have died. In the FS public sector, the mortality rate of people with CD4 counts $<350$ cells $/ \mu \mathrm{l}$ is $53 \%$ at 1 year, most of whom (87\%) did not start ARVs. ${ }^{15}$

Projections of patients starting ARVs in 2008 and 2009, based on the performance of the programme in the first 4 years, suggest that by 2009 only $39 \%$ of the newly ill patients with AIDS would access ARVs in the public sector. This figure is below the NSP's goal of 55\% of patients who need ARVs being able to access them by $2009 .{ }^{9}$ If the FS manages to achieve NSP goals, the cumulative number of people on ARVs would increase by about $150 \%$. The 7 large urban sites handling chronic ARV care for more than 1000 patients by the end of 2007 would increase patient load to more than 2500 . Subsequently, this increasing load of chronic care would soon see clinics exceed their capacity, a problem noted by other ARV sites in South Africa. ${ }^{16}$

Considering the shortage of human and financial resources, even modest projected increases for 2008 and 2009 may not be achievable, especially if the ARV roll-out is continued as a vertical programme. Of the 534 new posts created in the ARV programme, $38.8 \%$ were vacant by June 2008. This may be related to findings of a high level of burnout and compassion fatigue among ARV and PHC nurses in the FS. ${ }^{17}$

Funding of comprehensive HIV care in the FS increased by only $24 \%$ in the $2008 / 2009$ budget, and by $20 \%$ in the $2009 / 2010$ budget, whereas expected costs for drugs and laboratory tests (costs directly related to the number of people on ARVs and already taking up $35 \%$ of the budget) would have increased by $150 \%$ if there were over 44000 people on ARVs by the end of 2009. Although the public sector ARV programme in the FS is increasing the access to ARVs for people who need them, human and financial resources appear insufficient to achieve universal coverage, particularly if the vertical approach is continued.

Several models of broadening an initial vertical approach to achieve universal ARV access include: (i) down-referral, where ARV sites refer stable patients to obtain their continuing ARV supply at PHC clinics. ${ }^{18}$ This approach was mostly used as an afterthought to solve the problem of a saturated vertical service, but others see it as a gateway to integrate the provision of ARVs into PHC services; (ii) task shifting, a strategy supported by WHO, ${ }^{19}$ the essence of which is to equip and utilise lower cadres of health care workers to deliver comprehensive HIV care as a strategy to overcome shortages of highly skilled health care workers; and (iii) integration of HIV and ARV care into PHC services, a strategy implemented by 3 rural subdistricts in South Africa that have almost reached universal access. ${ }^{20}$

The FS DOH decided to broaden the programme by using a combination of task shifting (equipping nurses to initiate and repeat ARV prescriptions for uncomplicated patients) and integration of comprehensive HIV care into PHC services. The intervention commenced in early 2008 and is being implemented and evaluated as a pragmatic randomised controlled trial (STRETCH trial) in 31 of the current ARV sites, and is due to be completed in June 2010. ${ }^{21}$ The South African National DOH recently announced its accelerated AIDS plan with the aim of increasing access to antiretroviral therapy (ART). ${ }^{22}$ Task shifting and integration of ART into primary care services are central components of this plan. The experience of the STRETCH trial should provide important lessons in how to implement such a diagonal approach to expanding ART care and particularly how to equip and support nurses in primary care services to provide ART care. ${ }^{23}$

Our sincere thanks to Dr Eduan Kotze for help with data, and to staff in the FS office of the DOH, the districts, and the ARV sites for their assistance; the staff at the NHLS for assistance with data linkage for CD4 results; and Daleen Struwig, medical writer, Faculty of Health Sciences, University of the Free State, for technical and editorial preparation of the manuscript for publication.

Financial support was received in the form of a doctoral bursary from the SA National Research Foundation (KEU) and a Capacity Building Studentship from the UK MRC (SMI).

\section{References}

1. UNAIDS/WHO. Epidemiological Fact Sheets on HIV and AIDS: Core Data on Epidemiology and Response, South Africa. 2008 Update. Geneva: UNAIDS/ WHO, 2008. http://apps.who.int/globalatlas/predefinedReports/EFS2008/full/ EFS2008_ZA.pdf (accessed 2 April 2010).

2. Dorrington R, Johnson L, Bradshaw D, Daniel T. The Demographic Impact of HIV/AIDS in South Africa: National and Provincial Indicators for 2006. Cape Town: Centre for Actuarial Research, South African Medical Research Council and Actuarial Society of South Africa, 2006.

3. Shisana O, Rehle T, Simbayi L, et al. South African National HIV Prevalence, Incidence, Behaviour and Communication Survey 2008: A Turning Point Among Teenagers? Cape Town: HSRC Press, 2009.

4. Mukherjee J, Farmer P, Niyizonkiza D, et al. Tackling HIV in resource poor countries. BMJ 2003; 327: 1104-1106.

5. World Health Organization. Towards Universal Access: Scaling Up Priority HIV/AIDS Interventions in the Health Sector: Progress Report. Geneva: WHO Press, 2008.

6. Department of Health. Operational Plan for Comprehensive HIV and AIDS Care, Management and Treatment for South Africa 2003. Pretoria: South African Department of Health, 2003.

7. Atun RA, Bennett S, Duran A. When Do Vertical (Stand Alone) Programmes Have a Place in Health Systems? Copenhagen: WHO, 2008.

8. Ooms G, Van Damme W, Baker B, Zeitz P, Schrecker T. The diagonal approach to global fund financing: a cure for the broader malaise of health systems? Globalisation and Health 2008; 4: 6 .

9. Department of Health. National Strategic Plan 2007. HIV and AIDS and STI Strategic Plan for South Africa 2007-2011. Pretoria: South African Department of Health, 2007.

10. Van Rensburg D. The Free State's approach to implementing the comprehensive plan: notes by a participant outsider. Acta Academica Supplementum 2006: 4493.

11. Steyn F, Van Rensburg D, Engelbrecht M. Human resource for ART in the Free State public sector: recording achievements, identifying challenges. Acta Academica Supplementum 2006: 94-139.

12. Division of Revenues Schedule 5. Specific Allocation to Provinces Health (Vote 16)(a). Pretoria: SA Government Gazette. http://www.treasury.gov.za/ legislation/acts/2008/Division\%20of\%20Revenue\%20Act\%202\%20of\%202008. pdf (accessed 25 September 2008).

13. Actuarial Society of South Africa. 2003 AIDS and Demographic Model. ProvOutput_051129.zip. hsttp:www.actuarialsociety.org.za/Portals/1/ Documents/972db49b-9da4-472e-8557-2092cd69a92a.zip (accessed 8 September 2008). 
14. Fairall L, Zwarenstein M, Bateman E, et al. Effect of educational outreach to nurses on tuberculosis case detection and primary care of respiratory illness: pragmatic cluster randomised controlled trial. BMJ 2005; 331: 750-754.

15. Fairall L, Bachman M, Louwagie G, et al. Effectiveness of antiretroviral treatment in a South African program: a cohort study. Arch Int Med 2008; 168: 86-93.

16. Boulle A, Coetzee D. Anticipating future challenges to ART provision in South Africa: reflections on the Khayelitsha ART programme. Acta Academica Supplementum 2006: 241-255.

17. Van den Berg H, Bester C, Janse van Rensburg-Bonthuyzen E, et al. Burnout and Compassion Fatigue in Professional Nurses: A Study in PHC Facilities in the Free State, With Special Reference to the Antiretroviral Treatment Programme. Bloemfontein: Centre for Health Systems Research \& Development, University of the Free State, 2006.

18. Bennett B, Dlamini L, Mkhize E, Reid S, Barker P. The eight steps to successful down referral: opening the door to a PHC driven ARV program. http://www. ihi.org/IHI/Topics/DevelopingCountires/SouthAfrica/EmergingContent/ DownReferralPoster.htm (accessed 8 September 2008)
19. World Health Organization. Task Shifting: Rational Redistribution of Tasks Among Health Workforce Teams. Global Recommendations and Guidelines. Geneva: WHO Press, 2007.

20. Schneider H, Van Rensburg D, Coetzee D. Health Systems and Antiretroviral Access: Key Findings and Policy Recommendations. Round Table Conference Report. Bloemfontein: Centre for Health Systems Research \& Development, University of the Free State, 2007.

21. Fairall L, Bachman M, Zwarenstein M, et al. Streamlining tasks and roles to expand treatment and care for HIV: randomised controlled trial protocol. Trials 2008; 9: 21-26.

22. Gordhan P. Budget speech 2010 by the Minister of Finance 17 February 2010. http://www.doh.gov.za/docs/sp0217-f.html. (accessed 19 March 2010).

23. Colvin C, Fairall L, Lewin S, et al. Expanding access to ART in South Africa: The role of nurse-initiated treatment. S Afr Med J 2010; 100(4): 210-212.

Accepted 11 May 2010

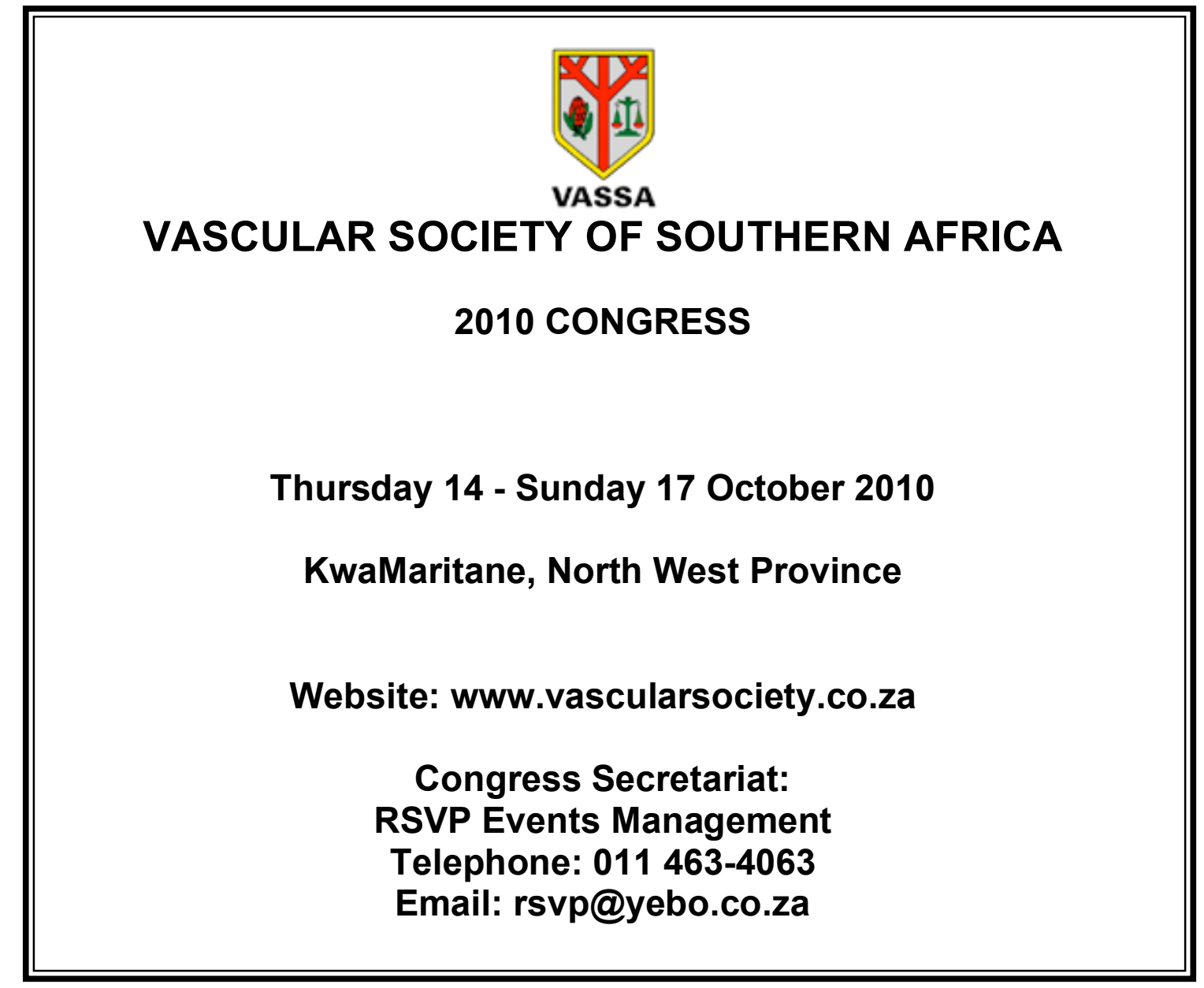

